\title{
Review \\ Clinical development of anti-RANKL therapy
}

Edward M Schwarz ${ }^{1}$ and Christopher T Ritchlin²

\begin{abstract}
1The Center for Musculoskeletal Research, University of Rochester Medical Center, Elmwood Avenue, Rochester, New York 14642, USA
2Department of Medicine, University of Rochester Medical Center, Elmwood Avenue, Rochester, New York 14642, USA
\end{abstract}

Corresponding author: Edward M Schwarz, edward_schwarz@urmc.rochester.edu

Published: 29 June 2007

This article is online at http://arthritis-research.com/content/9/S1/S7

(c) 2007 BioMed Central Ltd

Arthritis Research \& Therapy 2007, 9(Suppl 1):S7 (doi:10.1186/ar2171)

\begin{abstract}
The receptor activator of nuclear factor- $\mathrm{KB}$ ligand (RANKL), its cognate receptor RANK, and its natural decoy receptor osteoprotegerin have been identified as the final effector molecules of osteoclastic bone resorption. This has provided an ideal target for therapeutic interventions in metabolic bone disease. As described in previous reviews in this supplement, RANKL signaling is required for osteoclast differentiation, activation, and survival. Furthermore, in vivo inhibition of RANKL leads to immediate osteoclast apoptosis, and there are no in vivo models of bone resorption that are refractory to RANKL inhibition. Thus, the only step remaining in the development of a clinical intervention is the generation of a safe, effective, and specific drug that can inhibit RANKL in humans. Here we review the clinical development of denosumab (formerly known as AMG 162), which is a fully human $\mathrm{mAb}$ directed against RANKL. This discussion includes the breadth of 21 human studies that have led to the current phase 3 clinical trials seeking approval for use of this agent to treat postmenopausal women with low bone mineral density (osteoporosis) and patients with metastatic lytic bone lesions (multiple myeloma, and prostate and breast cancer).
\end{abstract}

\section{Introduction}

As discussed in previous reviews in this supplement, a specific inhibitor of receptor activator of nuclear factor- $\kappa \mathrm{B}$ ligand (RANKL) could be of great therapeutic value in the treatment of patients with a variety of metabolic and inflammatory bone disorders. Numerous biologic agents have been developed, including protein ligands (calcitonin [Miacalcin ${ }^{\mathrm{TM}}$; Novartis, East Hanover, NJ, USA], parathyroid hormone [Forteo $^{\mathrm{TM}}$; Eli Lilly, Indianapolis IN, USA], and bone morphogenetic protein-2 [Infuse ${ }^{\mathrm{TM}}$; Medtronic, Memphis, TN, USA]), soluble receptors (tumor necrosis factor [TNF] receptor-Fc [Enbrel ${ }^{\mathrm{TM}}$; Amgen, Thousand Oaks, CA, USA] and cytotoxic T-lymphocyte associated antigen-4 immunoglobulin [Orencia ${ }^{\mathrm{TM}}$; Bristol-Myers Squibb, New York, NY, USA]), and mAbs (anti-TNF [Remicade ${ }^{\mathrm{TM}}$; Centocor, Horsham, PA, USA and Humira ${ }^{\mathrm{TM}}$; Abbott, Abbott Park, IL,
USA] and anti-CD20 [Rituxan ${ }^{\mathrm{TM}}$; Genentech, San Francisco, CA, USA]). These have emerged as drugs of choice for many conditions as they are effective and, because they target a single molecular interaction, they are relatively safe. This specificity is largely due to the hundreds of millions of years of evolution (ligands and receptors) or more than $10^{12}$ DNA recombination events (mAbs) that occur to produce a highaffinity $\left(>10^{-9} \mathrm{M}\right)$ interaction with only one other protein in the body. Methods to achieve this kind of specificity with small molecule drugs are not available. Thus, even with rational drug design approaches based on atomic structures, undesirable interactions with other proteins that cause severe side effects often occur. Furthermore, some of these side effects only occur in humans, and so the first signals may only appear in clinical trials or postmarketing reports.

Development of a specific biologic agent for the treatment of human disease requires the evaluation of several different iterations and formulations. Indeed, this was the case for denosumab. Natural antagonists to RANKL were produced first because it was relatively easy to clone the cDNAs for RANK [1] and OPG [2] into expression vectors that would produce large amounts of the encoded proteins (receptor activator of nuclear factor- $\kappa B$ [RANK] and osteoprotegerin [OPG], respectively) in vitro. To facilitate their use in animal models, the Fc portion of the immunoglobulin heavy chain was fused to the amino-terminus of OPG (Fc-OPG) and the carboxyl-terminus of RANK (RANK-Fc) to generate effective recombinant proteins. The $\mathrm{Fc}$ domain allows for the dimerization required for high affinity to trimeric RANKL; it facilitates large-scale purification via protein $A$ or protein $G$ column chromatography; and it dramatically increases biodistribution and pharmacokinetics of the recombinant proteins in vivo. RANK-Fc and OPG-Fc proved to be very specific and effective inhibitors, and essentially all published preclinical studies to date have used these recombinant proteins [3].

$\mathrm{BMD}=$ bone mineral density $; \mathrm{mAb}=$ monoclonal antibody $\mathrm{NTX}=\mathrm{N}$-telopeptide; $\mathrm{ONJ}=$ osteonecrosis of the jaw; OPG = osteoprotegerin; PMO = postmenopausal osteoporosis; RA = rheumatoid arthritis; RANK = receptor activator of nuclear factor- $\mathrm{KB}$; RANKL = receptor activator of nuclear factor- $\mathrm{KB}$ ligand; $\mathrm{TNF}=$ tumor necrosis factor. 


\section{Clinical history of Fc-OPG}

Amgen Inc. (Thousand Oaks, CA, USA) performed the first clinical trial to evaluate the efficacy of RANKL inhibition using recombinant FC-OPG as a drug in postmenopausal women with osteoporosis [4]. This phase 1 study was designed to evaluate the effect of a single subcutaneous dose (placebo, $0.1,0.3,1.0$, or $3.0 \mathrm{mg} / \mathrm{kg}$ ) on bone resorption, and the primary outcome measures were biochemical markers of collagen catabolism (urinary N-telopeptide [NTX] and deoxypyridinoline). The highest dose yielded an approximate $80 \%$ decrease in NTX levels 4 days after dosing and significant effects lasted for $\mathbf{4 5}$ days. The study also evaluated the effects of Fc-OPG on osteoblasts by monitoring serum levels of bone-specific alkaline phosphatase (BSAP), which were largely unaffected by the drug. Because Fc-OPG was well tolerated by all patients and no serious adverse events were reported, the investigators concluded that this RANKL inhibitor is a viable candidate.

A subsequent phase 1 trial evaluated a different formulation of OPG known as AMGN-0007 in patients with multiple myeloma or breast carcinoma who exhibited radiologically confirmed lytic bone lesions [5]. Because ethical considerations prohibit the use of placebo in such cancer patients, a double dummy, active controlled study design was used, in which each patient received either a single subcutaneous injection of AMGN-0007 (0.1, 0.3, 1.0, or $3.0 \mathrm{mg} / \mathrm{kg}$ ) accompanied by intravenous placebo, or intravenous pamidronate $(90 \mathrm{mg})$ accompanied by subcutaneous placebo. Although the study found AMGN-0007 to be well tolerated and that its effects on bone resorption were similar to those of pamidronate, this was to be the last clinical trial with OPG. The reasons for this were described by Bekker and coworkers [6] and stem largely from the facts that AMG 162 (denosumab) is superior to Fc-OPG in that it yields greater decreases in bone turnover markers at lower doses and it has a longer duration of antiresorptive effect at equivalent doses. Those authors also cited two potential concerns with Fc-OPG therapy that do not apply to AMG 162. The first is the generation of anti-Fc-OPG antibodies, which might cross-react with endogenous Fc-OPG, neutralizing its activity. The second potential concern is binding of Fc-OPG to TNF-related apoptosis-inducing ligand [7], which could inhibit its role in tumor surveillance. Thus, it has been proposed that AMG 162 is safer and more efficacious than Fc-OPG as a therapeutic inhibitor of RANKL.

\section{Denosumab: a fully human mAb directed against RANKL}

Denosumab is a fully human mAb directed against RANKL and is currently under investigation in phase 3 clinical trials $[8,9]$. Denosumab was generated by immunizing the XenoMouse [10] with full-length human RANKL protein. This immunization produced a fully human $\lg _{1} m A b$, known as AMG 161, which recognized an epitope with a single amino acid difference between the mouse and human sequences
(Kostenuik PJ, personal communication). Because human $\lg _{1}$ can direct complement-dependent cytotoxicity or antibody-dependent cell cytotoxicity to target cells [11], there was concern that AMG 161 would have an undesirable toxic profile caused by death of RANKL-producing cells (stromal cells, osteoblasts, and T cells). Thus, it was converted to a noncytotoxic $\operatorname{lgG}_{2} \mathrm{mAb}$, known as AMG 162, which has an extremely high affinity (Kd approximately $10^{-12} \mathrm{M}$ ) for human RANKL. The fact that it does not recognize rodent RANKL has complicated preclinical development somewhat, in that the only relevant animal data were derived from studies conducted in cynomolgus monkeys [12].

\section{Clinical experience with denosumab}

Table 1 summarizes the 21 clinical trials to date that investigated the effects of denosumab in patients with postmenopausal osteoporosis (PMO), prostate cancer, breast cancer, multiple myeloma, solid tumors, and rheumatoid arthritis (RA). The initial phase 1 studies in PMO [6], and multiple myeloma or breast cancer [13] focused on pharmacokinetics and pharmacodynamics while monitoring adverse events. Both studies evaluated the effects of a single subcutaneous injection $(0.01,0.03,0.1,0.3,1.0$, or $3.0 \mathrm{mg} / \mathrm{kg}$ ) and monitored denosumab levels in serum and urine NTX levels. The results revealed that the drug effects had a rapid onset (within 12 hours) and were sustained in some patients for up to 6 months. In the trial conducted by Bekker and coworkers (in osteoporosis) [6] the maximum half-life was 32 days. In beast cancer and multiple myeloma the mean halflives were 46.3 and 33.3 days, respectively [13]. No drugrelated serious adverse events occurred in these phase 1 studies.

A phase 2 trial conducted to identify an ideal dose and treatment regimen for denosumab in $\mathrm{PMO}$ was recently completed [14], with the primary end-point being bone mineral density (BMD). Similar studies were conducted in patients with metastatic cancer, but the primary outcome was urine NTX $[15,16]$. Four other phase 2 trials are ongoing. These include a head-to-head comparison of denosumab with alendronate in $\mathrm{PMO}$ using bone structure as the primary outcome measure; a trial evaluating the efficacy of denosumab in treating multiple myeloma, in which complete and partial responses will be assessed; and an evaluation of the anti-RANKL effects of denosumab (60 or $180 \mathrm{mg}$ subcutaneously every 6 months) on cartilage degradation and bone erosion (assessed using plain radiographs and magnetic resonance imaging) in RA. The 6-month magnetic resonance imaging data from the RA trial were presented in November at the American College of Rheumatology annual meeting [17]. The results indicate that, compared with placebo, the $180 \mathrm{mg}$ group exhibited significantly less progression of bone erosions compared with placebo $(P<0.019)$. Although progression in the $60 \mathrm{mg}$ group was also numerically less than that with placebo, this did not reach significance $(P=0.247)$. These results are consistent with the finding that 


\section{Clinical trials investigating the effects of denosumab}

Study name or title on www.clinicaltrials.gov

Osteoporosis

A single dose, placebo-controlled study of AMG 162, a fully human mAb to RANKL, in postmenopausal women [6]

A randomized, double-blind, placebo-controlled, multidose phase 2 study to determine the efficacy, safety, and tolerability of AMG 162 in the treatment of postmenopausal women with low BMD [14]

A randomized, double-blind, placebo-controlled, dose-response study of AMG 162 (denosumab) in Japanese postmenopausal osteoporotic women

A multicenter, randomized, placebo-controlled, pilot microCT study to estimate the effect of treatment with denosumab (AMG 162) and alendronate sodium in postmenopausal women with low BMD

A study to evaluate AMG 162 in the treatment of postmenopausal osteoporosis A randomized, double-blind study to compare the efficacy of treatment with denosumab with that of alendronate sodium in postmenopausal women with low BMD

A randomized, double-blind study to evaluate safety and efficacy of transitioning therapy from alendronate to denosumab (AMG 162) in postmenopausal women with low BMD

A randomized, double-blind study to evaluate AMG 162 in the prevention of postmenopausal osteoporosis

An open-label, single arm extension study to evaluate the long-term safety of denosumab administration in postmenopausal women with low BMD

Bone metastases/multiple myeloma

A study of the biological RANKL inhibitor denosumab in subjects with multiple myeloma or bone metastases from breast cancer [13]

Randomized, active-controlled study of denosumab (AMG162) in breast cancer patients with bone metastasis previously treated with intravenous bisphosphonates [16]

A randomized trial of denosumab (AMG 162) versus intravenous bisphosphonates in cancer patients with bone metastases on established IV BP and evidence of elevated bone resorption [15]

An open-label, multicenter, phase 2 trial of denosumab in the treatment of relapsed or plateau-phase multiple myeloma

An open-label, multicenter, phase 2 safety and efficacy study of denosumab (AMG 162) in subjects with recurrent or unresectable giant cell tumor (GCT) of bone

A randomized, double-blind multicenter study of denosumab compared with zoledronic acid in the treatment of bone metastases in men with hormone-refractory prostate cancer

A randomized, double-blind, multicenter study of denosumab compared with zoledronic acid in the treatment of bone metastases in subjects with advanced breast cancer

Double-blind study of denosumab compared with zoledronic acid in the treatment of bone metastases in subjects with advanced cancer (excluding breast and prostate cancer) or multiple myeloma

A randomized, double-blind, placebo-controlled, multicenter phase 3 study of denosumab on prolonging bone metastasis-free survival in men with hormone refractory prostate cancer

Treatment-induced bone loss

A randomized, double-blind, placebo-controlled study to evaluate AMG 162 in the treatment of bone loss in patients undergoing androgen deprivation therapy for nonmetastatic prostate cancer

A randomized, double-blind, placebo-controlled study to evaluate AMG 162 in the treatment of bone loss in patients undergoing aromatase inhibitor therapy for nonmetastatic breast cancer

Rheumatoid arthritis

A randomized, double-blind, placebo-controlled, multidose phase 2 study to determine the efficacy, safety, and tolerability of AMG 162 in the treatment of rheumatoid arthritis [17]

Phase

$n$

Primary end-point

\begin{tabular}{|c|c|c|}
\hline phase 1 & 49 & $\begin{array}{l}\text { Pharmacokinetics } \\
\text { and } \\
\text { pharmacodynamics }\end{array}$ \\
\hline phase 2 & 412 & BMD \\
\hline phase 2 & - & BMD \\
\hline phase 2 & 240 & $\begin{array}{l}\text { Distal radius } \\
\text { measurements as } \\
\text { determined by } \\
\text { Xtreme CT }\end{array}$ \\
\hline phase 3 & 7,800 & Fracture \\
\hline phase 3 & 1,100 & BMD \\
\hline phase 3 & 500 & BMD \\
\hline phase 3 & 300 & BMD \\
\hline
\end{tabular}

Phase $3 \quad-\quad$ Safety

phase $1 \quad 54 \quad$ Pharmacokinetics and pharmacodynamics

phase 255 Urine NTX

phase $2 \quad 135 \quad$ Urine NTX

phase $2 \quad 100 \quad$ Complete

response/

partial response

phase $2 \quad 25 \quad$ Response rate based on imaging or tissue samples

phase $3 \quad 1,700 \quad$ Skeletal related events

phase $3 \quad 1,400 \quad$ Skeletal related events

phase $3 \quad 1,700 \quad$ Skeletal related events

phase $3 \quad 1,400 \quad$ Time to first occurence of bone metastasis or death from any cause

phase $3 \quad 1,400 \quad B M D$

phase $3 \quad 208 \quad$ BMD

$\begin{array}{lll}\text { phase } 2 & 227 & \text { Bone erosions }\end{array}$

CT, computed tomography; mAb, monoclonal antibody; NTX, N-telopeptide; RANKL, receptor activator of nuclear factor- $\mathrm{KB}$ ligand. 
markers of bone resorption (serum collagen C-terminal crosslinked peptide [CTX]-I and procollagen type 1 intact $\mathrm{N}$-terminal propeptide [P1NP]) and cartilage loss (urine CTX-II) were significantly decreased [18], and BMDs in the lumbar spine, total hip, and trochanter were significantly increased [19] in these RA patients on denosumab therapy.

Currently, there are 10 ongoing phase 3 clinical trials with denosumab for either PMO (60 mg subcutaneously every 6 months) or cancer (120 subcutaneously monthly). The largest is an international study in PMO in which drug effects on fracture reduction will be assessed. The other PMO trials will examine effects on BMD either as a head-to-head comparison with alendronate, after transition from bisphosphonate therapy, or as a prophylaxis in postmenopausal women. Additional pre-metastatic prostate cancer and breast cancer trials are underway that will analyze the effect of denosumab on bone loss related to androgen deprivation therapy and aromatase inhibitor therapy, respectively (Table 1). Another central question in the area of tumor metastasis is whether inhibition of bone remodeling will influence disease progression in terms of prolonging bone metastasis free survival in men with hormone refractory prostate cancer who have no bone metastases at baseline, or in terms of reducing skeletal related events (including pathologic fracture, radiation therapy to bone, surgery to bone, and spinal cord compression) in patients with bone metastases from prostate cancer, breast cancer, and solid tumors (Table 1).

\section{Potential side effects of denosumab therapy}

Three potential side effects have been identified based on information derived from the biology of RANKL, the literature on preclinical models of RANKL inhibition, and clinical experience of bisphosphonate therapy to date. Considerable effort has been invested in looking for signals related to these adverse events in the denosumab trials [20].

\section{Frozen bone}

The first potential side effect is so-called 'frozen bone', a process whereby complete inhibition of remodeling leads to accumulation of microfractures and brittle bone. In fact, concerns were raised regarding this complication in postmenopausal women receiving bisphosphonate therapy, based on findings from animal studies [21]. However, the fragility fractures that were predicted to occur in this population of patients, who have been treated with alendronate for more than 10 years, have not materialized. Frozen bone is less of an issue with denosumab in part because it is completely cleared from the body; this is in contrast to the more potent bisphosphonates, for which the half-life in bone may be 5 years or longer. The issue of decreased fracture healing has also been addressed in animal experiments [22]. These studies demonstrated that RANKknockout mice are able to heal their fractures, and therefore osteoclasts are not required to generate a union. The studies also indicated that discontinuing RANKL inhibition at the time of fracture in wild-type mice results in normal healing.

\section{Immunosuppression}

RANK-RANKL signaling was originally identified as a costimulatory pathway during T-cell activation [23], and RANK [24] and RANKL [25] knockout mice have altered lymphocyte development and lack lymph nodes. These facts have led to a concern regarding potential immunosuppression. Subsequent findings, however, have revealed that the role of RANK-RANKL co-stimulation in T-cell activation is only evident in the absence of CD40-CD40 ligand signaling [26]. It has also been shown that RANKL inhibition with OPG does not alter cellular or humoral immunity, or render mice susceptible to bacterial challenge [27]. Thus, although dendritic cells and T lymphocytes express these molecules, it would appear that they play a minor or redundant role in the mammalian immune response. Of course, close attention will be given to the incidence of infection and neoplasms in the larger phase 3 trials.

\section{Osteonecrosis of the jaw}

Osteonecrosis of the jaw (ONJ) has recently emerged as an adverse side effect of bisphosphonate therapy $[28,29]$. Currently, the nature of ONJ remains enigmatic, and its cause is a topic of great debate. Although it has been established that $94 \%$ of published cases occurred in cancer patients who received intravenous nitrogen-containing bisphosphonates, ONJ has also been reported in healthy women who take oral bisphosphonates for PMO. It is also known that $60 \%$ of cases are preceded by a dental surgical procedure, and so it has been proposed that the etiology of ONJ involves oversuppression of bone turnover combined with infection and possibly additional, as yet unidentified co-morbidities. Based on the serious nature of ONJ and our lack of knowledge on its etiology, the American Society for Bone and Mineral Research has commissioned a task force to investigate this problem [30]. Thus, until we have additional data, investigators should be aware of this potentially severe complication, and practice vigilant monitoring of patients who take all forms of anticatabolic therapy for bone loss.

\section{Advantages of denosumab over bisphosphonates}

Although the comparative ratios of risk to benefit for bisphosphonates and denosumab are currently unknown, there are three potential advantages of denosumab over bisphosphonates. As mentioned above, the first is that denosumab is completely cleared over a relatively short period of time, so that the effects on bone may not be longlasting. The second is that it could be the first anti-bone resorbing agent to halt focal erosions and osteolysis. Over the past two decades clinical trials designed to demonstrate the effectiveness of bisphosphonates on erosions in RA have repeatedly failed to show such an effect [31-33]. Interestingly, one of the more recent, well designed studies 
was able to demonstrate that etidronate therapy had a favorable effect on general bone metabolism (osteopenia), but it did not inhibit serum NTX levels or worsening of erosion scores in patients with RA [34]. The results from these trials are consistent with our preclinical studies, which showed that TNF protects osteoclasts from alendronate-induced apoptosis by stimulating $\mathrm{Bcl}-\mathrm{xL}$ expression through Ets-2 [35], while RANKL inhibition in the same setting of inflammation leads to rapid osteoclast apoptosis [36,37]. Thus, there is great expectation that denosumab will effectively prevent erosions in inflammatory arthritis and periprosthetic osteolysis.

Another disappointment of bisphosphonate therapy is the inability to use these agents effectively and safely in combination with anabolic agents. This was formally demonstrated in the teriparitide and alendronate trial conducted in patients with PMO [38]. Mechanistically, there are two prevailing explanations for this. The first relates to uncoupling of osteoclast-mediated bone resorption and new bone formation by osteoblasts. If failure of combination regimens results from uncoupling, then similar results to those with alendronate are expected with denosumab. On the other hand, if the lack of osteogenesis is due to osteoblast toxicity, such as that observed in preclinical models [39], which does not occur with RANKL inhibition $[36,40]$, then denosumab would be expected to work in combination with anabolic agents.

\section{Conclusion}

The discovery of the RANK, RANKL, and OPG pathway as the final effector of bone resorption has generated great expectations regarding the potential of a biologic antagonist for the treatment of patients with various metabolic and inflammatory bone disorders. The broad clinical trial experience to date indicates that denosumab may be a biologic agent that can effectively inhibit bone resorption with minimal side effects. The hope is that ongoing phase 3 clinical trials will demonstrate similar efficacy and safety profiles for denosumab therapy in $\mathrm{PMO}$ and metastatic cancer. Finally, looking to the future, it is not unreasonable to expect that other metabolic bone diseases, which have been refractory to bisphosphonates, will respond to this novel therapeutic approach.

\section{Competing interests}

During the past five years, EMS has received reimbursements, fees, and research funding from the sponsor (Amgen Inc.). No funding was received for preparation of this manuscript. EMS holds stock or shares in Amgen Inc. CTR is a consultant to Wyeth/Amgen and is an investigator on the AMG 162 RA trial.

\section{Acknowledgements}

The authors are supported by Public Health Service grants from $\mathrm{NIH}$, NIAMS (RO1s AR46545, AR48149, AR51469 AR/AG48697, and P50 AR54041).
This article is published as part of Arthritis Research \& Therapy Volume 9 Supplement 1, 2007: Basic science, rationale, background and future of denosumab: a RANK ligand inhibitor. The full contents of the supplement are available online at http://arthritis-research.com/supplements/9/S1.

Publication of the supplement has been supported by an unrestricted grant from Amgen Inc.

\section{References}

1. Anderson DM, Maraskovsky E, Billingsley WL, Dougall WC, Tometsko ME, Roux ER, Teepe MC, DuBose RF, Cosman D, Galibert L: A homologue of the TNF receptor and its ligand enhance T-cell growth and dendritic-cell function. Nature 1997, 390:175-179.

2. Simonet WS, Lacey DL, Dunstan CR, Kelley M, Chang MS, Luthy $\mathrm{R}$, Nguyen HQ, Wooden S, Bennett L, Boone T, et al.: Osteoprotegerin: a novel secreted protein involved in the regulation of bone density. Cell 1997, 89:309-319.

3. Boyle WJ, Simonet WS, Lacey DL: Osteoclast differentiation and activation. Nature 2003, 423:337-342.

4. Bekker PJ, Holloway D, Nakanishi A, Arrighi M, Leese PT, Dunstan CR: The effect of a single dose of osteoprotegerin in postmenopausal women. J Bone Miner Res 2001, 16:348-360.

5. Body JJ, Greipp P, Coleman RE, Facon T, Geurs F, Fermand JP, Harousseau JL, Lipton A, Mariette X, Williams CD, et al.: A phase I study of AMGN-0007, a recombinant osteoprotegerin construct, in patients with multiple myeloma or breast carcinoma related bone metastases. Cancer 2003, 97:887-892.

6. Bekker PJ, Holloway DL, Rasmussen AS, Murphy R, Martin SW, Leese PT, Holmes GB, Dunstan CR, DePaoli AM: A single-dose placebo-controlled study of AMG 162, a fully human monoclonal antibody to RANKL, in postmenopausal women. J Bone Miner Res 2004, 19:1059-1066.

7. Wiley SR, Schooley K, Smolak PJ, Din WS, Huang CP, Nicholl JK, Sutherland GR, Smith TD, Rauch C, Smith CA, et al.: Identification and characterization of a new member of the TNF family that induces apoptosis. Immunity 1995, 3:673-682.

8. Weiner LM: Fully human therapeutic monoclonal antibodies. $J$ Immunother 2006, 29:1-9.

9. Lonberg N: Human antibodies from transgenic animals. Nat Biotechnol 2005, 23:1117-1125.

10. Green LL: Antibody engineering via genetic engineering of the mouse: XenoMouse strains are a vehicle for the facile generation of therapeutic human monoclonal antibodies. J Immunol Methods 1999, 231:11-23.

11. Scallon BJ, Moore MA, Trinh H, Knight DM, Ghrayeb J: Chimeric anti-TNF-alpha monoclonal antibody CA2 binds recombinant transmembrane TNF-alpha and activates immune effector functions. Cytokine 1995, 7:251-259.

12. Kostenuik PJ: Osteoprotegerin and RANKL regulate bone resorption, density, geometry and strength. Curr Opin Pharma$\mathrm{col}$ 2005, 5:618-625.

13. Body JJ, Facon T, Coleman RE, Lipton A, Geurs F, Fan M, Holloway D, Peterson MC, Bekker PJ: A study of the biological receptor activator of nuclear factor-kappaB ligand inhibitor, denosumab, in patients with multiple myeloma or bone metastases from breast cancer. Clin Cancer Res 2006, 12: 1221-1228.

14. McClung MR, Lewiecki EM, Cohen SB, Bolognese MA, Woodson GC, Moffett AH, Peacock M, Miller PD, Lederman SN, Chesnut $\mathrm{CH}$, et al.: Denosumab in postmenopausal women with low bone mineral density. N Engl J Med 2006, 354:821-831.

15. Suarez T, Fizazi K, Rahim Y, Wilson JM, Fan M, Jun S, Lipton A: A randomized trial of denosumab (AMG 162) versus intravenous bisphosphonates in cancer patients with bone metastases on established IV BP and evidence of elevated bone resorption [abstract]. J Clin Onco/ 2006, 24:8562.

16. Lipton A, Alvarado C, De Boer R, Steger GG, Tonkin KD, Kinsey A, Fan M, Jun S: Randomized, active-controlled study of denosumab (AMG162) in breast cancer patients with bone metastasis previously treated with intravenous bisphosphonates [abstract]. J Clin Oncol 2006, 24:512.

17. Cohen SB, Valen P, Ritchlin CT, Schechtman J, Peterfy C, van der Heijde D, Zhou L, Newmark R, Tsuji W: RANKL inhibition with denosumab reduces progression of bone erosions in patients with rheumatoid arthritis: month 6 MRI results [abstract 2120]. Arthritis Rheum 2006, 54:S831. 
18. Lane NE, lannini M, Atkins C, Haraoui B, Zhou L, Tsuji W, Newmark R: RANKL inhibition with denosumab decreases markers of bone and cartilage turnover in patients with rheumatoid arthritis [abstract 451]. Arthritis Rheum 2006, 54: S225.

19. Dore R, Hurd E, Palmer W, Shergy W, Zhou L, Newmark R, Tsuji $W$ : Denosumab increases bone mineral density in patients with rheumatoid arthritis [abstract 488]. Arthritis Rheum 2006, $54: S 240$.

20. Whyte MP: The long and the short of bone therapy. $N$ Engl J Med 2006, 354:860-863.

21. Allen MR, Iwata K, Phipps R, Burr DB: Alterations in canine vertebral bone turnover, microdamage accumulation, and biomechanical properties following 1-year treatment with clinical treatment doses of risedronate or alendronate. Bone 2006, 39:872-879.

22. Flick LM, Weaver JM, Ulrich-Vinther M, Abuzzahab F, Zhang X, Dougall WC, Anderson D, O'Keefe RJ, Schwarz EM: Effects of receptor activator of NFkappaB (RANK) signaling blockade on fracture healing. J Orthop Res 2003, 21:676-684.

23. Wong BR, Rho J, Arron J, Robinson E, Orlinick J, Chao M, Kalachikov S, Cayani E, Bartlett FS III, Frankel WN, Lee SY, Choi $Y$ : TRANCE is a novel ligand of the tumor necrosis factor receptor family that activates c-Jun $\mathrm{N}$-terminal kinase in $\mathrm{T}$ cells. J Biol Chem 1997, 272:25190-25194.

24. Dougall WC, Glaccum M, Charrier K, Rohrbach K, Brasel K, De Smedt T, Daro E, Smith J, Tometsko ME, Maliszewski CR, et al:: RANK is essential for osteoclast and lymph node development. Genes Dev 1999, 13:2412-2424.

25. Kong YY, Yoshida H, Sarosi I, Tan HL, Timms E, Capparelli C, Morony S, Oliveira-dos-Santos AJ, Van G, Itie A, et al:: OPGL is a key regulator of osteoclastogenesis, lymphocyte development and lymph-node organogenesis. Nature 1999, 397:315323.

26. Bachmann MF, Wong BR, Josien R, Steinman RM, Oxenius A, Choi Y: TRANCE, a tumor necrosis factor family member critical for CD40 ligand-independent $T$ helper cell activation. $J$ Exp Med 1999, 189:1025-1031.

27. Stolina M, Guo J, Faggioni R, Brown H, Senaldi G: Regulatory effects of osteoprotegerin on cellular and humoral immune responses. Clin Immunol 2003, 109:347-354.

28. Woo SB, Hellstein JW, Kalmar JR: Systematic review: bisphosphonates and osteonecrosis of the jaws. Ann Intern Med 2006, 144:753-761.

29. Capsoni F, Longhi M, Weinstein R: Bisphosphonate-associated osteonecrosis of the jaw: the rheumatologist's role. Arthritis Res Ther 2006, 8:219.

30. Shane E, Goldring S, Christakos S, Drezner M, Eisman J, Silverman S, Pendrys D: Osteonecrosis of the jaw: more research needed. J Bone Miner Res 2006, 21:1503-1505.

31. Ralston SH, Hacking L, Willocks L, Bruce F, Pitkeathly DA: Clinical, biochemical, and radiographic effects of aminohydroxypropylidene bisphosphonate treatment in rheumatoid arthritis. Ann Rheum Dis 1989, 48:396-399.

32. Maccagno A, Di Giorgio E, Roldan EJ, Caballero LE, Perez Lloret $A$ : Double blind radiological assessment of continuous oral pamidronic acid in patients with rheumatoid arthritis. Scand J Rheumatol 1994, 23:211-214.

33. Eggelmeijer F, Papapoulos SE, van Paassen HC, Dijkmans BA, Valkema R, Westedt ML, Landman JO, Pauwels EK, Breedveld FC: Increased bone mass with pamidronate treatment in rheumatoid arthritis. Results of a three-year randomized, double-blind trial. Arthritis Rheum 1996, 39:396-402.

34. Valleala $\mathrm{H}$, Laasonen L, Koivula MK, Mandelin J, Friman C, Risteli J, Konttinen YT: Two year randomized controlled trial of etidronate in rheumatoid arthritis: changes in serum aminoterminal telopeptides correlate with radiographic progression of disease. J Rheumato/ 2003, 30:468-473.

35. Zhang Q, Badell IR, Schwarz EM, Boulukos KE, Yao Z, Boyce BF, Xing L: Tumor necrosis factor prevents alendronate-induced osteoclast apoptosis in vivo by stimulating $\mathrm{Bcl}-\mathrm{xL}$ expression through Ets-2. Arthritis Rheum 2005, 52:2708-2718.

36. Childs LM, Paschalis EP, Xing L, Dougall WC, Anderson D, Boskey AL, Puzas JE, Rosier RN, O'Keefe RJ, Boyce BF, et al.: In vivo RANK signaling blockade using the receptor activator of NF-kappaB:Fc effectively prevents and ameliorates wear debris-induced osteolysis via osteoclast depletion without inhibiting osteogenesis. J Bone Miner Res 2002, 17:192-199.

37. Li P, Schwarz EM, O'Keefe RJ, Ma L, Boyce BF, Xing L: RANK signaling is not required for TNFalpha-mediated increase in CD11(hi) osteoclast precursors but is essential for mature osteoclast formation in TNFalpha-mediated inflammatory arthritis. J Bone Miner Res 2004, 19:207-213.

38. Black DM, Greenspan SL, Ensrud KE, Palermo L, McGowan JA, Lang TF, Garnero P, Bouxsein ML, Bilezikian JP, Rosen CJ: The effects of parathyroid hormone and alendronate alone or in combination in postmenopausal osteoporosis. $N$ Engl J Med 2003, 349:1207-1215.

39. Iwata K, Li J, Follet H, Phipps RJ, Burr DB: Bisphosphonates suppress periosteal osteoblast activity independently of resorption in rat femur and tibia. Bone 2006, 39:1053-1058.

40. Kostenuik PJ, Capparelli C, Morony S, Adamu S, Shimamoto G, Shen V, Lacey DL, Dunstan CR: OPG and PTH-(1-34) have additive effects on bone density and mechanical strength in osteopenic ovariectomized rats. Endocrinology 2001, 142: 4295-4304. 